\title{
Contribution of Advection Process in SOC Model Causing the Appearance of Lognormal Distribution in Emission
}

\author{
Chatief Kunjaya, Putra Mahasena, Kiki Vierdayanti \\ and Stefani Herlie
}

Fac. Mathematics and Natural Sciences, Institut Teknologi Bandung, INDONESIA

\begin{abstract}
Emission variability from Black Hole objects like Seyfert Galaxies, Quasars etc. shows a irregular variation with various amplitude and time scale. Further analysis of the emission shows the appearance of the lognormal distribution. Such distribution cannot be explained by conventional Self Organized Criticality (SOC) Model. In this paper a modified SOC model with cellular automaton mechanism combined with advection process is introduced. Simulation shows that lognormal distribution can be resulted, while the other characteristics of PSD and structure function of the original SOC model can be maintained. Incorporation of the advection process is the key factor for the appearance of lognormal distribution.
\end{abstract}

Keywords. Accretion, accretion Disks

\section{Introduction}

One of the key for understanding the black hole objects like quasars, AGN etc. is their variability. The light variation from these objects is neither periodic nor random, with various time scales. Their power spectral density show a power law tendency. To explain this behaviour, several models has been introduced. Shot noise model was proposed by Terrel (1972). Starburst, disk instability, microlensing has also been discussed as the possible cause of the variability (e.g., Hawkins (2004)). One of the promising model was the self organized criticality (SOC) model which explained that the fluctuation in the light curve is caused by clumpy accretion in the accretion disk surrounding black hole objects (e.g., Mineshige et al. (1994)). However, recent observation of some black hole objects show the existence of multiplicative process in the light fluctuation, which is reflected in its lognormal flux distribution (e.g. Gaskell (2004)). The underlying process in the original SOC model is additive, therefore it is not possible to yield lognormal distribution. In this work, we show that combining advection process into the SOC model can yield lognormal flux distribution.

\section{Adding Advection Process to the SOC Model}

In the SOC model, accretion disk is divided into rings and sectors, the intersection of a ring and a sector is called a cell. The outermost cells receive mass input from the surrounding materials in random amount. Some part of the mass in each cell can fall into several adjacent inner cells through avalanche process when it reach a critical mass. Besides avalanche process there is diffusion process which move small mass from a randomly chosen cell in each ring to its adjacent inner cell. In the avalanche and diffusion, some mass move closer to the central black hole and convert potential energy into heat and radiation. For each time step, all off the energy release from avalanche and diffusion 


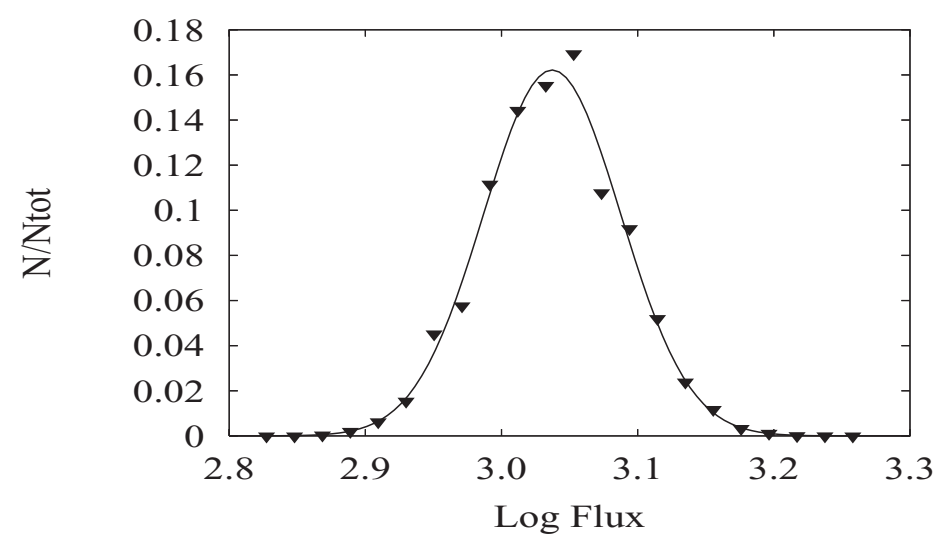

Figure 1. Flux distribution for advection domination with $a=0.1$, solid line is the theoretical lognormal function, while the solid triangle points are from simulation

is summed to yield the total emission from the disk. There is only additive process in the emission creation, therefore it is expected that the flux distribution will be normal. How can we explain the lognormal flux distribution like those found by Gaskell (2004)?

In the idea of ADAF (e.g., Ichimaru (1977), Abramowicz et al. (1988)), when some mass move closer to inner part of the disk, most of the dissipated energy is not directly radiated into the space, but will be stored in the disk and be brought into inner part of the disk along with the mass. The accumulation of energy will make the avalanche and diffusion in the next time step occur in higher energy content and consequently radiates more energy into space. This will create a multiplicative effect in emission creation and yield the lognormal distribution in the observed flux.

We simulate the fluctuative light curve from accretion disks using the idea, using parameter $a$ as the fraction of the dissipated energy emitted into space. In other words, $(1-a)$ is the measure of the contribution of advection process. The resulted light curve was then analized to check its PSD and Structure Function whether it is still agree with the usual characteristic of black hole objects. After confirming it, then the flux distribution was fitted with a lognormal function by adjusting the parameters of the function.

\section{Result}

By adding advection process into the SOC model we can create simulated light curve. The flux distribution show the lognormal characteristics and can be fitted with a lognormal function. This means the lognormal flux distribution can still be obtained from SOC model. This model can also yield normal flux distribution or intermediate between normal and lognormal by adjusting advection parameter $(1-a)$.

\section{References}

Abramowicz M. A., Czerny, B., Lasota, J. P., \& Szuszkiewicz, E. 1988, ApJ, 332, 646 Gaskell, C. M. 2004, ApJ, 612, L21

Hawkins, M. R. S. 2004, Baltic Astronomy, 13, 642

Ichimaru, S. 1977, $A p J, 214,840$

Mineshige, S., Ouchi, M. B., \& Nishimori, H. 1994, PASJ, 46, 97

Terrel, N. J. 1972, ApJL, 174, L35 